\title{
Prism Adaptation and Aftereffect: Specifying the Properties of a Procedural Memory System
}

\author{
Juan Fernández-Ruiz ${ }^{1}$ and Rosalinda Díaz \\ Laboratorio de Neuropsicología \\ Departamento de Fisiología \\ Universidad Nacional Autónoma de México \\ 04510 México D.F., México
}

\begin{abstract}
Prism adaptation, a form of procedural learning, is a phenomenon in which the motor system adapts to new visuospatial coordinates imposed by prisms that displace the visual field. Once the prisms are withdrawn, the degree and strength of the adaptation can be measured by the spatial deviation of the motor actions in the direction opposite to the visual displacement imposed by the prisms, a phenomenon known as aftereffect. This study was designed to define the variables that affect the acquisition and retention of the aftereffect. Subjects were required to throw balls to a target in front of them before, during, and after lateral displacement of the visual field with prismatic spectacles. The diopters of the prisms and the number of throws were varied among different groups of subjects. The results show that the adaptation process is dependent on the number of interactions between the visual and motor system, and not on the time spent wearing the prisms. The results also show that the magnitude of the aftereffect is highly correlated with the magnitude of the adaptation, regardless of the diopters of the prisms or the number of throws. Finally, the results suggest that persistence of the aftereffect depends on the number of throws after the adaptation is complete. On the basis of these results, we propose that the system underlying this kind of learning stores at least two different
\end{abstract}

\footnotetext{
${ }^{1}$ Corresponding author.
}

parameters, the contents (measured as the magnitude of displacement) and the persistence (measured as the number of throws to return to the baseline) of the learned information.

\section{Introduction}

Prism adaptation, a form of procedural learning, is a phenomenon in which the motor system adapts to new visuospatial coordinates imposed by prisms that displace the visual field horizontally. Once the prisms are withdrawn, the degree and strength of the adaptation can be measured by the aftereffect, the spatial deviation of the motor actions in the direction opposite to the visual displacement imposed by the prisms. This phenomenon has been studied since the late nineteenth century when Von Helmholtz published his seminal work in optics (Von Helmholtz 1962). During the mid-1960s, Held (1965) demonstrated that prism adaptation depends on the interaction between the motor and the visual systems and that such interaction normally induces a plastic change in the brain. Most research in prism adaptation during the last decades has been directed at identifying the plasticity locus, particularly whether it occurs in the motor system, the sensory system, or both systems (Bossom 1965; Harris 1965; Welch 1974; Kornheiser 1976).

Prism adaptation, however, can be studied from a different perspective. It can be used as a model for the analysis of the acquisition of procedural or nondeclarative knowledge. Tulving (1993) has defined the procedural memory system as an action system whose operations are expressed in the form of skilled behavioral and cognitive procedures independent of any cognition. Similarly, Squire (1993) has suggested that nondeclarative

LEARNING \& MEMORY 6:47-53 @ 1999 by Cold Spring Harbor Laboratory Press ISSN1072-0502/99 \$5.00

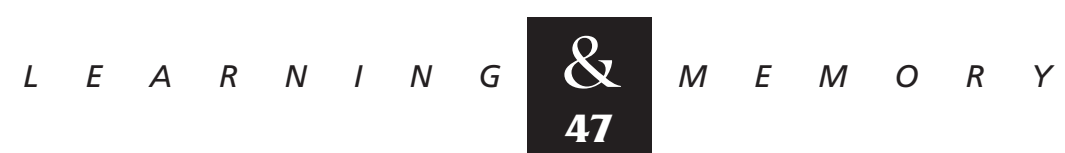


learning takes place when experience accumulates in behavioral change without affording conscious access to any memory content. Prism adaptation is an example of this kind of learning that can be more specifically classified as motor learning. Viewed from this perspective, prism adaptation of fers an important advantage for studying motor learning because it can dissociate performance from learning and memory. In a typical experiment, the initial motor performance of a naive subject is evaluated. The behavioral modification elicited by the use of the prisms provides an estimate of the learning rate. Upon removal of the prisms, the aftereffect provides a measure of the persistence of the memory.

Although the aftereffect is the principal indication that adaptation has occurred, many of its properties have not yet been determined. For instance, it has been established that no aftereffect develops in the absence of any visuomotor interaction (Held 1965). However, the effect of varying the number of interactions between the motor and visual systems on the strength of the aftereffect has not been examined rigorously. Similarly, the endurance of the aftereffect has not been studied carefully, presumably because the aftereffect has been considered exclusively as an indication that an adaptation has occurred, and not as an independent phenomenon. Recently, Martin et al. (1996a,b) implemented a different approach to the classical prism experiment. The investigators asked the subjects to throw clay balls at a target $2 \mathrm{~m}$ in front of them while wearing the prisms. Using this approach, we report the effects of different training conditions during prism adaptation on the degree of learning, the aftereffect, and the persistence of the memory.

\section{Materials and Methods}

\section{SUBJECTS}

A total of 100 unpaid healthy adult volunteers with no history of neurological injury was divided into 10 groups of 10 subjects each. The subjects were naive to the purpose of the experiment. In each group, five subjects were male and five were female. All subjects were right-handed, and all gave informed consent before the experiments in accordance with the Declaration of Helsinki.

\section{PROCEDURE}

In these experiments we followed the throw- ing technique described by Martin et al. (1996a,b). Throwing is a ballistic movement, in that there is no correction of the movement sequence once the movement has started (Ghez 1991). Subjects viewed the target binocularly through 10-, 20-, or 30-diopter Fresnel 3M Press-on plastic lenses (3M Health Care, Specialties Division, St. Paul, MN). The subjects threw clay balls at a target (a $10 \times 10$ $\mathrm{cm}$ cross drawn on a large sheet of parcel paper) centered at shoulder level $2 \mathrm{~m}$ in front of them. The subjects stood without changing their foot position during performance of the task, the head was unrestrained, and no directions were given about trunk, shoulder, or head/neck posture.

\section{GENERAL DESIGN OF EXPERIMENTS}

Each experiment followed the three phases described previously (Martin et al. 1996a,b). A baseline throwing performance was obtained by having the subjects throw 25 balls at the target before they donned prisms (PRE condition). During the whole experiment, the subjects were instructed to throw where they saw the target. The position at which the balls made an impact on or around the target was marked immediately after each throw. After donning prisms, the subjects were instructed to throw a variable number of balls (see below) with the same arm and in the same way (PRI condition), and the results were marked as described above. After removing the prisms, the subjects threw 25 more balls with the same arm and in the same way as before (POS condition). Subjects had an unobstructed view of the target during the entire session but were instructed not to look down at their hands as they collected the balls from a tray next to them during throws. The location of the impacts was plotted sequentially by trial number (abscissa) versus horizontal displacement (in $\mathrm{cm}$ ) from a vertical line passing through the target center (ordinate). Impacts to the left of the target were plotted as negative values and impacts to the right as positive values (Martin et al. 1996a,b).

In the first experiment, three groups of subjects were tested, and all of them threw 25 balls in each of the three conditions. The first group wore 10-diopter lenses, the second group 20-diopter lenses, and the third group 30-diopter lenses. In the second experiment six groups of subjects were tested, and all of them threw 25 balls in the PRE and POS conditions and wore 30-diopter lenses. In the PRI condition, however, the six groups threw $0,3,6,9,13$, or 25 balls, respectively. Subjects in

$$
\begin{array}{llllllllllllllll} 
& E & A & R & N & I & N & G & \mathbf{Q} \\
\mathbf{4 8} & M & E & M & O & R & Y
\end{array}
$$


the group that threw no balls were told to remain standing in a relaxed manner for 2 min with their eyes open before the POS condition started. Finally, in the third experiment, another group of subjects donned 30 diopter lenses and threw 50 balls during the PRI condition.

Two additional measures were calculated from the collected data. First, an adaptation measure was obtained by subtracting the distance to the center of the ball's impact on the final throw while wearing the prisms from that on the initial throw while wearing them. Second, an aftereffect measure was defined as the distance from the center of the first throw after removing the prisms.

\section{STATISTICAL ANALYSIS}

The results of the first and second experiment were analyzed using two-way repeated measure ANOVA with Student-Newman-Keuls post hoc analysis for multiple comparisons. Two-way ANOVA was used to compare multiple groups in a single condition (i.e., POS data for groups with different numbers of throws during the PRI condi- tion). One-way ANOVA was used to compare the number of throws needed to reach adaptation among the different diopter groups. The group in the third experiment was compared with the last group of the second experiment using both twoway repeated measure ANOVA with Student-Newman-Keuls post hoc analysis and Student's $t$-test for the total accumulated distance during the third phase of the experiment. A correlation was run between adaptation and aftereffect using Pearson's correlation analysis.

\section{Results}

ADAPTATION TO 10-, 20-, AND 30-DIOPTER PRISMS

Figure 1 shows the horizontal location of the points of impact before (PRE), during (PRI), and after (POS) prism adaptation in relation to the target for the three prisms $(10,20$, and 30 diopters) used in experiment 1 . An initial analysis was done to determine if there were differences between PRE, PRI, and POS within each diopter condition. Two-way repeated measure ANOVA analysis revealed a significant difference between PRE versus

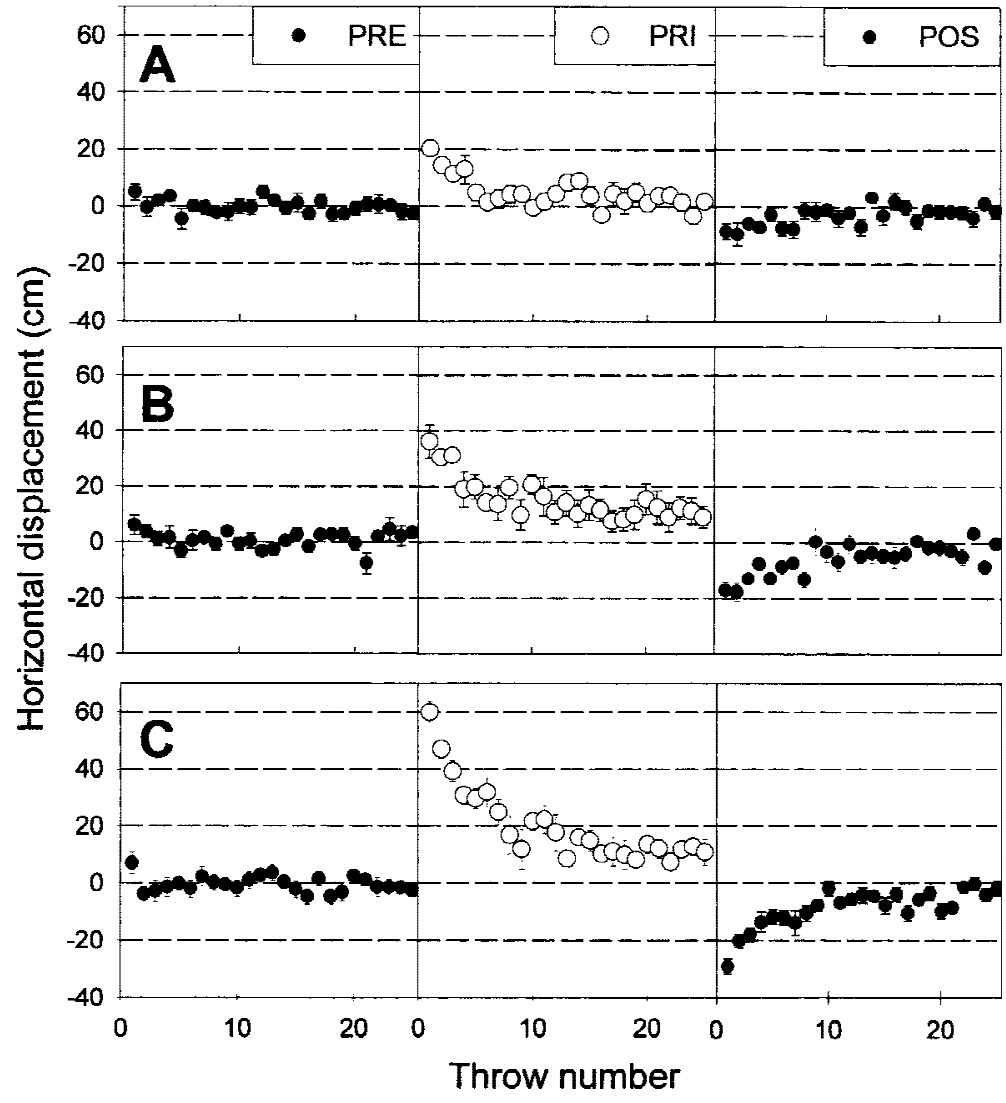

Figure 1: Horizontal displacement $(\mathrm{cm})$ of the impacts across 25 throws in three different conditions. (A) Donning 10-diopter prisms; (B) 20-diopter prisms; $(C)$ 30-diopter prisms. (PRE) Throws before donning prisms; (PRI) throws while wearing prisms; (POS) throws after removing the prisms. Data represent mean \pm S.E.M. 
PRI for the three sets of prisms (10, 20, and 30 diopters $)(P<0.05)$. The same analysis also revealed a significant difference between PRI and POS, as well as between PRE and POS for all three set of prisms $(P<0.05)$ for all cases. For example, in the group with the smallest differences, the 10diopter group, there was a statistically significant difference between PRE and PRI $[F(1,480)=29.9$, $P<0.01], \quad$ PRE and POS $[F(1,480)=9.975$; $P<0.01]$, and PRI and POS $[F(1,480)=89.22$, $P<0.01]$. The post hoc analyses (Student-Newman-Keuls) within this group showed differences between the PRE and PRI scores for the initial four throws and between PRE and POS scores for the initial two throws $(P<0.05)$.

To know the effect of wearing prisms with different diopters, two-way repeated measure ANOVA was run on the POS condition between all three diopters groups. The ANOVA showed a significant difference in the POS condition of the 10-, $20-$, and 30-diopters groups $[F(2,48)=8.61$; $P<0.01]$, and also revealed a statistically significant interaction between diopters and throws $[F(48,675)=1.88 ; P<0.01]$.

The initial distance of the impacts from the target while wearing the prisms was proportional to both the diopter of the prisms and the aftereffect magnitude. In addition, the aftereffect magnitude was also proportional to the diopter of the prisms.

A one-way ANOVA showed that the number of throws needed to reach adaptation (PRI condition) was different among the three groups $[F(2,747)=10.3, P<0.01]$. The mean throw at which the 30-diopters group reached the value of their maximum adaptation level (mean of the last 5 throws during PRI) was 12.2, the 20-diopters group reached it at 9 , and the 10-diopters group did it at the sixth throw. An all-pairwise multiple comparison procedure (Student-Newman-Keuls method) showed significant differences $(P<0.05)$ among all three groups.

\section{AFTEREFFECT FOR GROUPS WITH DIFFERENT ADAPTATION LEVELS}

Figure 2 shows the horizontal location of the points on impact for PRE, PRI, and POS in relation to the target for the six groups with different numbers of throws $(0,3,6,9,13$, and 25 , respectively) during the PRI condition in experiment 2 (Fig. 2). Two-way ANOVA revealed no differences among the six PRE conditions. The same analysis showed statistical differences in the POS condition across the different groups $[F(5,144)=7.22 ; P<0.001]$. A Student-Newman-Keuls analysis of the POS condition of the six groups showed differences $(P<0.05)$ among the 13-throws group and the first four groups $(0,3,6$, and 9$)$, and between the 25throws group and the first four groups. No differences were found among the first four groups $(0$, 3, 6, and 9). Statistical analysis revealed a signifi-

Figure 2: Horizontal displacement $(\mathrm{cm})$ of the impacts across throws in six different conditions. Zero, 3, 6, 9, 13 and 25 throws during the PRI condition $(A, B, C$, $D, E$, and $F$, respectively). All subjects donned 30-diopter prisms during PRI condition. Data represent means \pm S.E.M.
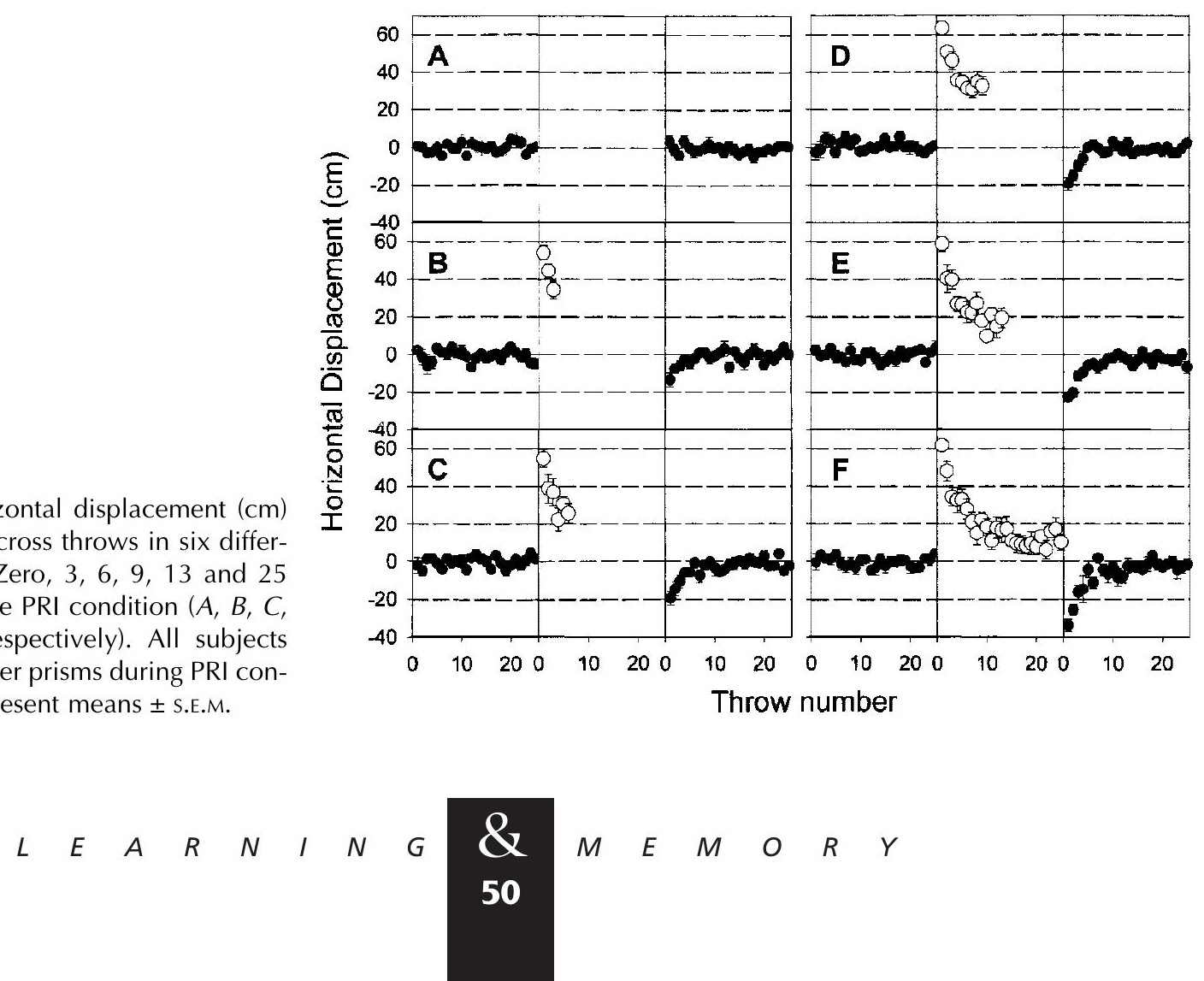
cant difference between PRE versus POS $(P<0.05)$ for all groups except the one that threw 0 balls during the PRI condition.

\section{CORRELATION BETWEEN ADAPTATION} AND AFTEREFFECT

As shown in Figure 3, there was a linear correlation $(r=-0.978, P<0.001)$ between adaptation (distance of the initial impact minus distance of the final impact during PRI) and aftereffect (distance from the target of the initial impact during POS) for eight groups (10-, 20-, and 30-diopters groups, and 3-, 6-, 9-, 13-, and 25-throws groups). The 0 PRI group was excluded from this analysis, as it had no initial adaptation level. Figure 3 also shows that the adaptation always had a larger magnitude than the aftereffect, regardless of the condition tested.

\section{THE EFFECT OF THROWING MORE BALLS AFTER} REACHING THE MAXIMUM AFTEREFFECT DISTANCE

Figure 4 (A, bottom) shows the horizontal location of the points of impact for PRE, PRI, and POS for a group that threw 50 balls during the PRI condition but threw 25 balls during PRE and POS conditions. The analysis showed a statistical difference $(P<0.05)$ between groups 50 and 25 for the POS condition (Fig. 4A). Student's $t$-test for the sum of the accumulated distance during the POS con-

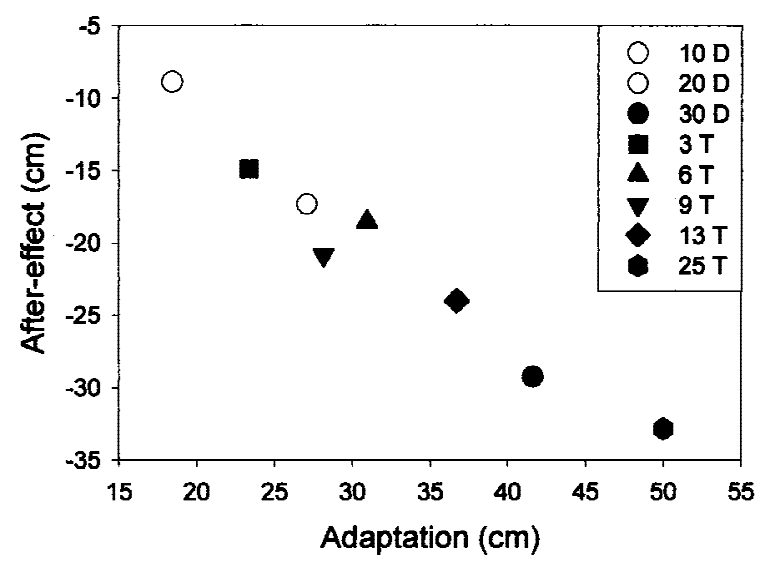

Figure 3: Correlation between adaptation and aftereffect for eight groups. (10D) 10-diopter group; (20D) 20diopter group, and (30D) 30-diopter group; (3 T) 3-Throw group; (6 T) 6-throw group; (9 T) 9-throw group; (13 T) 13-throw group; (25 T) 25-throw group. Pearson's correlation coefficient $(r=-0.978 ; P<0.001$. dition (25 throws in each group) (Fig. 4B) showed that the group that threw 50 balls during the PRI condition had a significantly larger accumulated distance (the sum of the distances of the 25 POS throws) than the group that threw 25 balls during the PRI condition $(t=-2.41, P<0.05)$, despite being equal on the initial aftereffect throw.

THE RATE OF CHANGE OF THE ORIGINAL AND THE RECENTLY ACQUIRED CALIBRATION

The PRI and POS data were best fitted by the reciprocal quadratic function of the form $f=1 / a+b x+c x^{2}$ (correlation coefficient $=$ 0.994). The PRI coefficient data obtained were $a=0.012775904, b=0.0057503569$, and $c=$ -0.00010987634 . The POS coefficient data were $a$ $=0.012775956, b=0.0057503284$, and $c=$ -0.00010987497 . To obtain the rate of change, the following derivative function was used: $f^{\prime}=-b+$ $2 c x /\left(a+b x+c x^{2}\right)^{2}$. To compare the rate of change for PRI and POS, the same absolute magnitude values were used. The derivative analysis showed a faster rate of change for the initial throws. The derivative values for the three initial adaptation throws were $-16.30,-9.34$, and -6.03 , respectively. The aftereffect values were -48.23 , -13.32 , and -6.17 , respectively.

\section{Discussion}

Our results lead to a number of conclusions regarding the properties of prism adaptation and, by extension, to the mechanisms of this type of procedural learning. First, the greater the prism diopters, the greater the initial throw displacement and the more throws needed to reach asymptotic level. Therefore, the motor system does not make an evaluation of the total displacement and recalibrate the throws instantly. If the system could make automatic evaluations of the visuomotor mismatch, then adaptation to the three prisms would take the same number of throws, which is not the case. Martin et al. (1996b) reported a subject who tried to hit the target using a cognitive strategy but whose motor performance followed the same pattern of adaptation described here. The results raise the question of why the system does not make bigger changes per throw. It is possible that the system neural substrate wiring could be designed for fine tuning, allowing only a maximum change per throw. Kitazawa et al. (1995) demonstrated

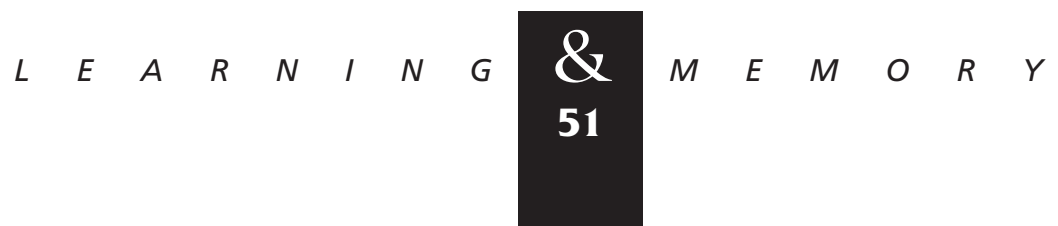


Figure 4: (A) Effect of throwing 25 (top) or 50 (bottom) balls during the PRI condition on the aftereffect. Accumulated horizontal displacement during the 25 POS throws (aftereffect) in both groups. Data represent mean \pm S.E.M. $(*) P<0.05$.
A

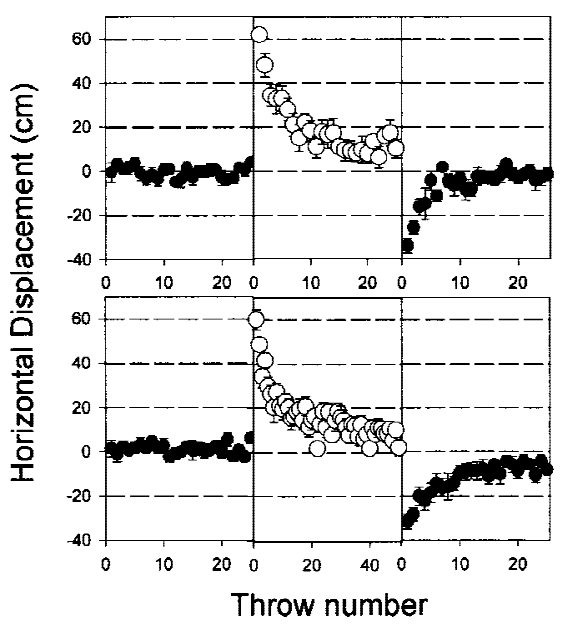

B

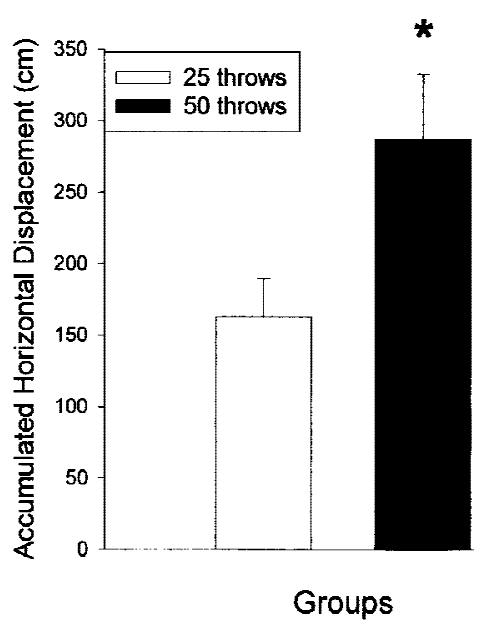

that in some conditions it is possible to find an error correction rate within the adaptation process that is an $\sim 9 \%$ decrease of the preceding error.

Second, the aftereffect depends on the number of interactions between the visual and the motor systems and not on the time spent wearing the prisms (for 0 interactions there is no aftereffect) (Prablanc et al. 1975; Martin et al. 1996b). The results show that as few as three interactions between the visual and the motor systems can produce robust aftereffects. The fast modification of the previously set visuomotor coordination suggests that the system is under constant maintenance, as proposed initially by Held (1965) and more recently by Thach et al. (1992).

Third, under our experimental conditions, the aftereffect did not have the same magnitude as the adaptation (Cohen 1966; Foley and Maynes 1969; Hardt et al. 1971; Taub and Goldberg 1973; Redding and Wallace 1988; Kitazawa et al. 1995; Martin et al. 1996a,b). The results show that it is not enough for the adaptation to reach an asymptote to obtain an aftereffect of the same magnitude as the adaptation. This implies that the aftereffect does not simply mirror the adaptation processes and that there are some unknown factors affecting one process and not the other (Kelso et al. 1975; Melamed et al. 1979; Melamed and Arnett 1984; Redding et al. 1985). For example, Jakobson and Goodale (1989) found that making subjects aware of the visual displacement by providing them with explicit information about the prisms led to reduced levels of adaptation.

Fourth, once the adaptation process reaches an asymptote, more interactions between the mo- tor response and visual feedback affect only the adaptation's persistence. That is, once the visuomotor system becomes recoordinated, the new calibration becomes strengthened with further practice (Fig. 4). It seems logical to suppose that the strengthening of the new calibration is subjected to the same lability or maintenance as the original calibration, so it would be expected that the rate of disappearance of the aftereffect once the prisms are withdrawn would be faster than or the same as the rate of modification of the original coordination. The derivative analysis of the functions that best fitted the PRI and POS data confirmed a faster decrease for the aftereffect curve than for the adaptation curve, suggesting a greater lability for the new acquired visuospatial coordination. Recently, Shadmehr and Holcomb (1997) suggested that functional stability in a motor skill may be achieved by a shift in the neural representation of the internal model from prefrontal regions of the cortex to the premotor, posterior parietal, and cerebellar cortex and that such a shift occurs within $\sim 6 \mathrm{hr}$ after completion of the motor skill practice. In the experiments described here, the new motor memory was achieved in seconds, as throwing 25 balls takes $<1 \mathrm{~min}$. This difference in time scale suggests the existence of at least two different processes during motor skill learning (for a recent discussion regarding multiple processes during motor skill learning, see Karni 1996).

In summary, we have shown that the aftereffect is not the simple mirror image of the adaptation process. Instead, some of its properties, such as its magnitude and its persistence, depend on different aspects of training.

$$
\begin{array}{llllllllllllllll}
L & E & A & R & N & I & N & G & \boldsymbol{Q} \\
\mathbf{5 2} & M & E & M & O & R & Y
\end{array}
$$




\section{Acknowledgments}

This work was supported in part by Universidad Nacional Autonoma de Mexico DGAPA grant IN214997. We thank Jacqueline E. Mishner, Juan Carlos López García, and Mortimer Mishkin for their helpful discussions and for their critical reading of the manuscript, and E. Javier Elizondo for his helpful mathematical assistance.

The publication costs of this article were defrayed in part by payment of page charges. This article must therefore be hereby marked "advertisement" in accordance with 18 USC section 1734 solely to indicate this fact.

\section{References}

Bossom, J. 1965. The effect of brain lesions on prism-adaptation in monkey. Psychon Sci. 2: 45-46.

Cohen, H.B. 1966. Some critical factors in prism-adaptation. Am. J. Psychol. 79: 285-290.

Foley, J.E. and F.J. Maynes. 1969. Comparison of training methods in the production of prism adaptation. J. Exp. Psychol. 81: 151-155.

Ghez, C. 1991. The control of movement. In Principles of neural science (ed. E.R. Kandel, J.H. Schwartz, and T.M. Jessell), pp. 533-547. Appleton \& Lange, Norwalk, CT.

Hardt, M.E., R. Held, and M.J. Steinbach. 1971. Adaptation to displaced vision: A change in the central control of sensorimotor coordination. J. Exp. Psychol. 89: 229-239.

Harris, C.S. 1965. Perceptual adaptation to inverted, reversed, and displaced vision. Psychol. Rev. 72: 419-444.

Held, R. 1965. Plasticity in sensory-motor systems. Sci. Am. 213: 84-94.

Jakobson, L.S. and M.A. Goodale. 1989. Trajectories of reaches to prismatically-displaced targets: Evidence for "automatic" visuomotor recalibration. Exp. Brain Res. 78: $575-587$.

Karni, A. 1996. The acquisition of perceptual and motor skills: A memory system in the adult human cortex. Brain Res. Cogn. Brain Res. 5: 39-48

Kelso, J.A., E. Cook, M.E. Olson, and W. Epstein. 1975. Allocation of attention and the locus of adaptation to displaced vision. J. Exp. Psychol. 75: 237-245.

Kitazawa, S., T. Kohno, and T. Uka. 1995. Effects of delayed visual information on the rate and amount of prism adaptation in the human. J. Neurosci. 15: 7644-7652.

Kornheiser, A.S. 1976. Adaptation to laterally displaced vision: A review. Psychol. Bull. 83: 783-816.

Martin, T.A., J.G. Keating, H.P. Goodkin, A.J. Bastian, and W. T. Thach. 1996a. Throwing while looking through prisms. I. Focal olivocerebellar lesions impair adaptation. Brain 119: $1183-1198$. 1996b. Throwing while looking through prisms. II. Specificity and storage of multiple gaze-throw calibrations. Brain 119: 1199-1211.

Melamed, L.E. and W.B. Arnett. 1984. The effect of familial sinistrality on perceptual learning. Neuropsychologia 22: 495-502.

Melamed, L.E., P. Beckett, and M. Halay. 1979. Individual differences in the visual component of prism adaptation. Perception 8: 699-706.

Prablanc, C., A. Tzavaras, and M. Jeannerod. 1975. Adaptation of the two arms to opposite prism displacements. Q. J. Exp. Psychol. 27: 667-671.

Redding, G.M., S.E. Clark, and B. Wallace. 1985. Attention and prism adaptation. Cognit. Psychol. 17: 1-25.

Redding, G.M. and B. Wallace. 1988. Components of prism adaptation in terminal and concurrent exposure:

Organization of the eye-hand coordination loop. Percept. Psychophys. 44: 59-68.

Shadmehr, R. and H.H. Holcomb. 1997. Neural correlates of motor memory consolidation. Science 277: 821-825.

Squire, L.R. 1993. Declarative and nondeclarative memory: Multiple brain systems supporting learning and memory. In Memory concepts. Basic and clinical aspects (ed. P. Andersen, O. Hvalby, O. Paulsen, and B. Hökfelt), pp. 3-25. Elsevier Science Publishers, New York, NY.

Taub, E. and I.A. Goldberg. 1973. Prism adaptation: Control of intermanual transfer by distribution of practice. Science 180: $755-757$.

Thach, W.T., H.P. Goodkin, and J.G. Keating. 1992. The cerebellum and the adaptive coordination of movement. Annu. Rev. Neurosci. 15: 403-442.

Tulving, E. 1993. Human memory. In Memory concepts. Basic and clinical aspects (ed. P. Andersen, O. Hvalby, O. Paulsen, and B. Hökfelt), pp. 27-45. Elsevier Science Publishers, New York, NY.

von Helmholtz, H. 1962. In Helmholtz's treatise on physiological optics, Vol. 3, Sect. 2. Translated from the Third German Ed. (ed. J.P.C. Southall), pp. 242-270. Dover Press, New York, NY.

Welch, R.B. 1974. Research on adaptation to rearranged vision: 1966-1974. Perception 3: 367-392.

Received June 2, 1998; accepted in revised form January 19, 1999.

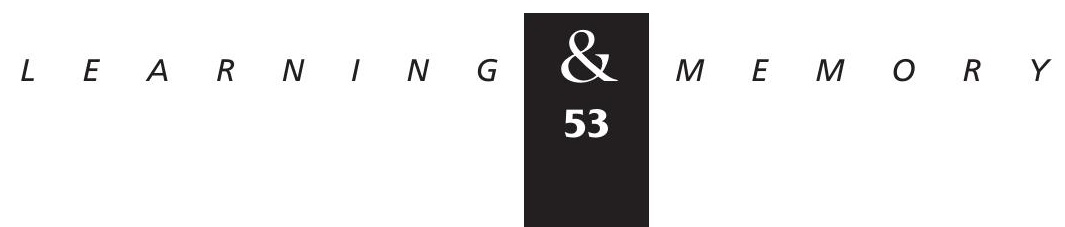




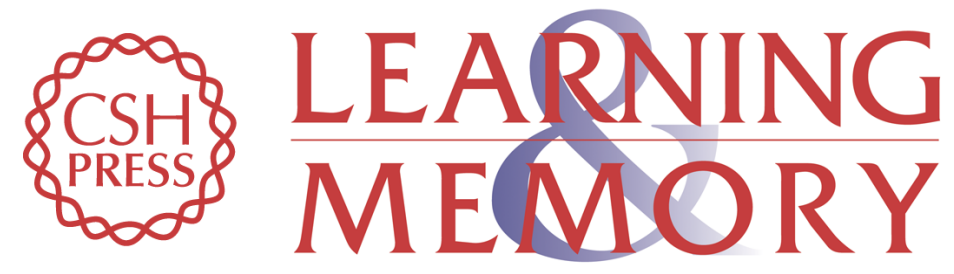

\section{Prism Adaptation and Aftereffect: Specifying the Properties of a Procedural Memory System}

Juan Fernández-Ruiz and Rosalinda Díaz

Learn. Mem. 1999, 6:

Access the most recent version at doi:10.1101/lm.6.1.47

References This article cites 21 articles, 3 of which can be accessed free at: http://learnmem.cshlp.org/content/6/1/47.full.html\#ref-list-1

License

Email Alerting Receive free email alerts when new articles cite this article - sign up in the box at the Service top right corner of the article or click here. 